\title{
Artigo original \\ Fatores associados ao comportamento sedentário em escolares de 9-12 anos de idade
}

\author{
Amanda Santos \\ Alynne Christian Ribeiro Andaki \\ Universidade Federal do Triângulo Mineiro \\ Paulo Roberto dos Santos Amorim \\ Universidade Federal de Viçosa \\ Edmar Lacerda Mendes \\ Universidade Federal do Triângulo Mineiro
}

\begin{abstract}
Resumo-São recentes os estudos que investigaram impactos do comportamento sedentário (CS) sobre a saúde, principalmente em crianças. O objetivo foi investigar fatores associados ao CS em escolares do município de Uberaba, MG. Trata-se de estudo epidemiológico, transversal, com amostra probabilística $(n=649)$. Foram coletadas e consideradas variáveis independentes: medidas antropométricas, pressão arterial, exames bioquímicos, tempo gasto em atividade física (AF). CS foi considerado variável dependente, caracterizado pelo tempo de tela (TT), e verificado fatores associados pela regressão de Poisson. Meninas apresentaram valores maiores de dobras cutâneas, triglicerídeos e HDL-c e meninos, maior concentração de glicose sanguínea, atenderam mais à recomendação de 60 min de AF/dia e maior CS. O TT $\geq 2 \mathrm{~h} /$ dia foi prevalente em $76,9 \%$ da amostra. CS não foi associado a variáveis sociodemográficas, de saúde e comportamentais em crianças de 9-12 anos de idade. Mais pesquisas são necessárias para elucidar as inter-relações complexas entre TT e fatores associados.
\end{abstract}

Palavras-chaves: estilo de vida sedentário, criança, associação

\begin{abstract}
Factors associated with sedentary behavior in 9- to 12-year-old school children." Studies that investigated the impacts of sedentary behavior (SB) on health are recent, especially in children. The aim of this study was to investigate factors associated with SB in a Brazilian school in the city of Uberaba, State of Minas Gerais. This is an epidemiological study using cross-sectional probability sample $(\mathrm{n}=649)$. We collected and considered as independent variables: anthropometric measurements, blood pressure, biochemical, time spent in physical activity (PA). SD was considered the dependent variable, characterized by screen time (ST), and verified associated factors using Poisson regression analysis. Girls had higher skinfold, triglycerides and HDL-c, and boys have higher blood glucose concentration, attended more to the recommendation $60 \mathrm{~min} \mathrm{PA} /$ day and higher SB. The TT $\geq 2 \mathrm{~h}$ /day was prevalent in $76,9 \%$ of the sample. SB was not associated with sociodemographic, health nor behavioral variables in 9- to 12-year-old school children. More research is necessary to identify the complex interrelationships between ST and associated factors.
\end{abstract}

Keywords: sedentary lifestyle, children, association

Resumen - "Factores asociados con la conducta sedentaria en escolares de 9-12 años de edad." Son recientes los estudios que han investigado los efectos del conducta sedentaria (CS) en la salud, especialmente en niños. El objetivo fue investigar los factores asociados con CS en escolares Uberaba-MG. Es epidemiológico, sección transversal y muestra aleatoria, $(\mathrm{n}=649)$. Se recogieron y consideraba como variables independientes: medidas antropométricas, presión arterial, pruebas bioquímicas, tiempo de actividad física (AF). CS fue considerado variable dependiente, caracteriza por el tiempo de pantalla (TT), y verificado factores asociados mediante regresión Poisson. Niñas fueron mayores del pliegue cutáneo, triglicéridos y HDL-c y los niños, la concentración de glucosa en la sangre, asistieron más la recomendación de $60 \mathrm{~min}$ de $\mathrm{AF} /$ día y superiores CS. TT $\geq 2 \mathrm{~h} /$ día era frecuente en $76,9 \%$ de muestra. CS no se asoció con variables sociodemográficas, de salud y comportamiento en los niños de 9-12 años de edad. Se necesitan más investigaciones para dilucidar las complejas relaciones entre TT y factores asociados.

Palabras claves: estilo de vida sedentario, niño, asociación 


\section{Introdução}

Comportamento sedentário (CS) pode ser referido como "tempo sentado" e tem sido retratado em atividades que não aumentam o gasto energético substancialmente acima do nível de repouso, por exemplo, permanecer sentado, assistir televisão ou se dedicar a outras formas de entretenimento baseadas em tela (Pate, O'Neill, \& Lobelo, 2008; Tremblay, Leblanc, Janssen, et al., 2011).

O tempo gasto em CS tem sido associado a resultados negativos à saúde como composição corporal desfavorável (te Velde et al., 2012; Tremblay, Leblanc, Kho, et al., 2011), à biomarcadores de doenças cardiovasculares e metabólicas como a síndrome metabólica (SM) (Della Manna, Damiani, \& Setian, 2006; Edwardson et al., 2012; Kang et al., 2010) e menor capacidade cardiorrespiratória (Aggio, Ogunleye, Voss, \& Sandercock, 2012; Hardy, Dobbins, Denney-Wilson, Okely, \& Booth, 2009; Harrison, Burns, McGuinness, Heslin, \& Murphy, 2006).

O CS pode contribuir para o surgimento da SM, termo utilizado para descrever conjunto de fatores de risco de doença cardiovascular, caracterizado por índices antropométricos, dislipidemias e pressão arterial alterada (PAA) (Della Manna et al., 2006; Sardinha \& Magalhães, 2012). Indivíduos que dedicam grande parte do dia ao CS possuem risco aumentado de $73 \%$ em desenvolver SM (Edwardson et al., 2012). A prevalência de SM é mais acentuada em crianças e adolescentes obesos (Tavares, Yokoo, Rosa, \& Fonseca, 2010), e, por outro lado a adoção de um estilo de vida saudável, ainda na infância e adolescência, contribui para minimizar o surgimento precoce da doença cardiovascular (Della Manna et al., 2006).

Foram observadas associações entre CS e baixa aptidão cardiorrespiratória, deslocamento passivo para escola e obesidade, que persistem após ajuste para a AF (Danielsen et al., 2011; Ekelund et al., 2006; Sandercock \& Ogunleye, 2012). De tal modo, o CS e AF devem ser considerados construtos diferenciados (Ekelund et al., 2006; Pate, Mitchell, Byun, \& Dowda, 2011)

O CS é um construto relativamente recente e merecedor de mais averiguações como foco principal. Investigar os fatores potencialmente modificáveis associados ao CS pode embasar estratégias e metodologias eficientes para intervenções, a fim de reduzir o tempo de exposição e minimizar os danos à saúde. Neste sentido, o objetivo do presente estudo foi investigar os fatores associados ao CS em escolares de 9-12 anos de idade do município de Uberaba, MG.

\section{Método}

\section{Tipo de pesquisa e procedimentos de aleatorização da amostra}

O estudo foi do tipo epidemiológico, observacional, de delineamento transversal e amostra representativa composta por escolares de ambos os sexos, regularmente matriculados no $5^{\circ}$ ano do ensino fundamental das escolas de Uberaba, MG.

O estudo obteve aprovação do Comitê de Ética em Pesquisa com Seres Humanos da Universidade Federal do Triângulo Mineiro (Protocolo CEP/UFTM 1710) e autorização para sua realização nas unidades de ensino da Secretaria Municipal de Educação e Cultura de Uberaba, MG.

A amostragem empregada foi do tipo probabilística, estratificada e proporcional. Os dados utilizados para o cálculo amostral foram acessados no Censo Escolar 2011 Educacenso. Encontravam-se matriculados no $5^{\circ}$ ano de ensino fundamental 4412 alunos. A amostra mínima calculada foi de 353 crianças, erro tolerável de $5 \%$ e nível de confiança de $95 \%$, calculado pelo programa EpiInfo 7. Acrescentou-se $20 \%$ para minimizar as variáveis de confundimento e $10 \%$ para possíveis perdas, totalizando amostra de 459 crianças.

\section{Avaliação antropométrica}

A avaliação antropométrica contou com mensuração da estatura por meio de estadiômetro portátil (Welmy, Santa Bárbara do Oeste, Brasil) e da massa corporal pela balança eletrônica digital (Plena, modelo ICE, São Paulo, Brasil). O índice de massa corporal (IMC) foi categorizado de acordo com Cole, Bellizzi, Flegal e Dietz (2000).

As dobras cutâneas tricipital (DCT) e subescapular (DCSe) foram obtidas por adipômetro (Lange, Cambridge, USA). Para estimativa da composição corporal foram utilizadas as equações de Slaughter et al. (1988) e o índice de adiposidade foi categorizado de acordo com o percentual de gordura corporal (\%GC) (T. G. Lohman, 1987). O perímetro da cintura (PC) foi medido no ponto anatômico de menor curvatura do abdome entre a crista ilíaca e as costelas (Timothy G. Lohman, Roche, \& Martorell, 1988) e considerado PC alterado (PCalt) $\geq$ percentil 75 para idade e sexo da amostra. Todas as medidas foram feitas em triplicata e considerado o valor médio das três mensurações.

\section{Pressão arterial}

A pressão arterial (PA) foi aferida pelo método indireto com técnica auscultatória a partir do esfigmomanômetro de coluna de mercúrio portátil (Unitec, modelo Plus, São Paulo, Brasil). Após repouso de 5 a $10 \mathrm{~min}$, a PA foi aferida três vezes com intervalo mínimo de 1 min entre as aferições e foi considerado o valor médio das duas últimas. A classificação da pressão arterial foi determinada com base nos pontos de corte específicos para crianças, de acordo com o percentil para idade, estatura e sexo. Foram diagnosticadas com pressão arterial alterada (PAA) as crianças que apresentaram a pressão arterial sistólica (PAS) ou diastólica (PAD) acima dos valores de pressão arterial referentes ao percentil arterial 90, seguindo as normas propostas pelas VI Diretrizes Brasileiras de Hipertensão (Nobre, 2010). 


\section{Análise bioquímica}

Amostras de $8 \mathrm{~mL}$ de sangue foram coletadas em tubos a vácuo (BD Vacutainer, São Paulo, Brasil), por profissional habilitado, utilizando material descartável, após jejum de 12 a 14 horas das crianças. As amostras foram centrifugadas a $3.400 \mathrm{rpm}$ durante oito minutos para separação do soro e plasma dos demais componentes do sangue, e avaliadas no analisador bioquímico semiautomático (Bioplus, modelo BIO200F, São Paulo, Brasil). Para análise de glicemia (método GOD-Trinder), triglicerídeos e HDL-c (método colorimétrico) foram utilizados kits comerciais (LABTEST, Sistema Diagnóstico Ltda. Lagoa Santa, Brasil) de aplicação manual e método de ponto final. Os exames com valores alterados foram repetidos para confirmação do teste.

\section{Síndrome metabólica}

A SM foi diagnosticada pela presença de pelo menos três dos seguintes fatores: obesidade ( $\mathrm{PC} \geq$ percentil 75 da amostra para idade e sexo), hiperglicemia $(\geq 110 \mathrm{mg} / \mathrm{dL})$, hipertrigliceridemia ( $\geq 100 \mathrm{mg} / \mathrm{dL})$, baixo nível de HDL-c ( $<$ $50 \mathrm{mg} / \mathrm{dL})$ e PAA ( $>$ percentil 90 ajustado para idade, estatura e sexo) (de Ferranti et al., 2004).

\section{Recomendação de atividade física}

A prática de atividade física moderada a vigorosa (AFMV), o tempo de envolvimento em minutos, e intensidade foram obtidos a partir do instrumento Lista de Atividades Físicas (LAF), versão brasileira do Physical Activity Checklist Interview (Sallis et al., 1996), por meio de entrevistas individuais, motivando as crianças a recordarem o seu dia anterior com perguntas: Que horas você acordou ontem? Brincou de alguma coisa pela manhã? Teve aula de educação física ontem? Depois da aula, você ficou em casa? Foi para rua brincar? Que horas você dormiu?

Foi considerado satisfatório o envolvimento em AFMV $\geq 60 \mathrm{~min} / \mathrm{dia}$ (Department of Health, 2004; U.S. Department of Health and Human Services, 2008; World Health Organization, 2010).

\section{Comportamento sedentário e aspectos comportamentais}

Questionário intitulado Hábitos de Vida (Andaki, 2010) foi respondido por pais ou responsáveis sobre tempo de tela (TT) e aspectos comportamentais de seus filhos como: meio de deslocamento para escola, prática de AF fora da escola e tempo de sono.

Para estimar o TT, as perguntas utilizadas foram: Quanto tempo diariamente seu filho passa em frente à televisão? Quanto tempo diariamente seu filho passa em frente ao computador (dele, de amigos ou em lan house)? Quantas horas por dia seu filho passa jogando videogame? As opções de respostas foram: $0 \mathrm{~min}, 30 \mathrm{~min}, 1 \mathrm{~h}, 2 \mathrm{~h}, 3 \mathrm{~h}, 4 \mathrm{~h}$ ou $+4 \mathrm{~h}$, em dias de semana e finais de semana. Os aspectos comportamentais foram avaliados por meio das seguintes perguntas: Seu filho frequenta alguma escolinha esportiva? Com resposta afirmativa ou negativa. Qual o meio de transporte utilizado para o deslocamento de seu filho até a escola? Com opções de respostas: carro, van escolar, moto, ônibus, a pé, bicicleta. Quantas horas aproximadamente seu filho dorme por noite? Com respostas entre: menos de $4 \mathrm{~h}, 4$ a $5 \mathrm{~h}, 5$ a $6 \mathrm{~h}$, 6 a $7 \mathrm{~h}, 7$ a $8 \mathrm{~h}$, mais de $8 \mathrm{~h}$.

CS foi entendido como tempo gasto pelo indivíduo sentado por longos períodos frente à televisão, videogame ou computador, caracterizado como TT. Para classificar crianças que apresentam CS foi utilizado TT $\geq 2$ horas/dia.

\section{Classificação econômica}

Por meio do questionário da Associação Brasileira de Empresas de Pesquisa/ABEP (2011), foi utilizado o critério de classificação econômica do Brasil, de forma a estimar o poder de compra das pessoas e famílias urbanas, permitindo a estratificação em classes econômicas.

\section{Categorização e agrupamento das variáveis}

Foi considerado variável dependente o CS dicotomizado em TT ( $<2$ horas e $\geq 2$ horas). O modelo hierárquico foi construído com os seguintes blocos e variáveis independentes: (1) Aspectos demográficos e socioeconômicos: sexo (masculino e feminino); idade cronológica (anos) e classe econômica (A-B e C, D-E); (2) Aspectos de saúde: IMC categorizado (normal e excesso de peso), classificação da gordura corporal (ótimo e alto), SM (sim e não); (3) aspectos comportamentais: AFMV $(<60 \mathrm{~min} / \mathrm{dia} \mathrm{e} \geq 60 \mathrm{~min} / \mathrm{dia})$, deslocamento para escola (ativo - a pé ou de bicicleta; passivo - van escolar, carro ou moto), tempo de sono por noite $(<8 \mathrm{~h}$ e $\geq 8 \mathrm{~h}$ ), frequência em escolinha esportiva (sim e não).

\section{Análise estatística}

A normalidade dos dados foi checada pelo teste de Kolgomorov-Smirnov. De acordo com a distribuição, comparações entre os sexos foram analisadas utilizando-se os testes para amostras independentes t de Student e MannWhitney. Os dados descritivos foram apresentados em média e desvio-padrão, quando paramétricos, e em mediana e diferença inter-quartis, quando não-paramétricos. Análises bivariadas $(p<0,20)$ e multivariáveis foram realizadas por meio da regressão de Poisson, que apresenta como medida de associação a razão de prevalência (RP). O nível de significância adotado foi de 5\%. A estruturação do banco de dados foi realizada no software Epidata versão 3.1 e análises estatísticas no programa SPSS 20.0. 


\section{Resultados}

\section{Caracterização da amostra}

Participaram do estudo 649 crianças, 338 do sexo feminino $(52,1 \%)$, com valores medianos de idade de 10,0 $\pm 1,0$ anos, massa corporal $=33,5 \pm 12,0 \mathrm{~kg}$, estatura $=1,4 \pm 0,1 \mathrm{~m}$ e IMC $=17,3 \pm 4,7 \mathrm{~kg} / \mathrm{m} 2$.

As crianças apresentaram composição corporal, avaliadas por meio das dobras cutâneas, de $11,1 \%$ baixo \%GC, $45,1 \%$ ótimo \%GC, 14,6\% moderadamente alto e $29,1 \%$ alto. Prevalência de sobrepeso, obesidade e SM foi observada em $11,3 \%, 5,6 \%$ e $12,3 \%$ da amostra, respectivamente. Hiperglicemia e hipertrigliceridemia foram observadas em $0,2 \%$ e $19,8 \%$ da amostra, respectivamente. Por outro lado, reduzidos níveis de HDL-c apresentaram prevalência de $77,2 \%$. PAA e PCalt foram prevalentes em $8,3 \%$ e $25,2 \%$ dos casos. E ainda, alcançaram a recomendação de AFMV $\geq 60$ $\mathrm{min} /$ dia $55,1 \%$ das crianças. Comparações entre os sexos estão presentes na Tabela 1 .

\section{Aspectos socioeconômicos e hábitos de vida}

O CS foi prevalente em $76,9 \%$ da amostra. O tempo mediano gasto de TT foi de 225,0 $\pm 195,0 \mathrm{~min} / \mathrm{dia}$. Assistir $\mathrm{TV}$ foi a forma de entretenimento baseado em tela mais utilizado (150,0 $\pm 195,0 \mathrm{~min} / \mathrm{dia})$. A classe econômica $\mathrm{C} 1$ foi predominante $(35,3 \%)$, seguida pela C2 $(21,6 \%)$, B2 $(18,1 \%)$, $\mathrm{D}(17,3 \%), \mathrm{B} 1(6,7 \%), \mathrm{A} 2(0,5 \%)$ e $\mathrm{E}(0,5 \%)$.

\section{Regressão de Poisson}

Não foram encontradas interações entre CS e variáveis

Tabela 1. Caracterização das variáveis antropométricas, de saúde e comportamentais dos escolares de 9-12 anos de idade do município de Uberaba, MG, Brasil, 2012.

\begin{tabular}{|c|c|c|c|c|c|c|}
\hline \multirow{2}{*}{ Variáveis } & \multicolumn{3}{|c|}{ Meninas } & \multicolumn{3}{|c|}{ Meninos } \\
\hline & $\mathrm{n}$ & Mediana & Diferença inter-quartis & $\mathrm{n}$ & Mediana & Diferença inter-quartis \\
\hline Idade cronológica & 304 & 10 & 1,0 & 277 & 10 & 1,0 \\
\hline \multicolumn{7}{|l|}{ Antropometria } \\
\hline Massa corporal (kg) & 301 & 33,8 & 12,3 & 274 & 33,2 & 11,6 \\
\hline $\operatorname{IMC}\left(\mathrm{kg} / \mathrm{m}^{2}\right)$ & 301 & 17,3 & 4,7 & 274 & 17,3 & 4,7 \\
\hline $\mathrm{PC}(\mathrm{cm})$ & 301 & 60,1 & 9,7 & 273 & 59,8 & 10,3 \\
\hline \multicolumn{7}{|l|}{ Composição corporal } \\
\hline $\mathrm{DCT}(\mathrm{mm})$ & 301 & $14,7 *$ & 8,8 & 274 & 12,7 & 8,3 \\
\hline $\mathrm{DCSe}(\mathrm{mm})$ & 301 & $11 *$ & 10,7 & 274 & 7,7 & 9,0 \\
\hline$\Sigma 2 \mathrm{DC}(\mathrm{mm})$ & 301 & $26^{*}$ & 20,5 & 274 & 20 & 18,7 \\
\hline$\% \mathrm{GC}$ & 301 & $23,3 *$ & 13,5 & 274 & 18,8 & 14,3 \\
\hline \multicolumn{7}{|l|}{ Bioquímicas } \\
\hline TRI (mg/dL) & 231 & $73 *$ & 36,5 & 204 & 66 & 36,8 \\
\hline HDL-c (mg/dL) & 234 & $40 *$ & 14,0 & 200 & 43 & 14,0 \\
\hline \multicolumn{7}{|l|}{ Pressão arterial } \\
\hline Sistólica (mmHg) & 297 & 100 & 20,0 & 273 & 100 & 19,5 \\
\hline Diastólica $(\mathrm{mmHg})$ & 297 & 65 & 12,0 & 273 & 63 & 12,0 \\
\hline \multicolumn{7}{|l|}{ Atividade física } \\
\hline AFMV (minutos) & 287 & $60 *$ & 71,0 & 274 & 70 & 90,0 \\
\hline CMTp (METs) & 287 & $286^{*}$ & 434,4 & 274 & 426,9 & 625,2 \\
\hline \multicolumn{7}{|l|}{ Comportamento sedentário } \\
\hline TV-Sem (min/dia) & 218 & 120 & 120,0 & 198 & 120 & 120,0 \\
\hline TV - Fds (min/dia) & 219 & 180 & 180,0 & 189 & 180 & 180,0 \\
\hline Computador - Sem (min/dia). & 214 & 30 & 60,0 & 192 & 15 & 60,0 \\
\hline Computador - Fds (min/dia) & 214 & 30 & 120,0 & 187 & 30 & 120,0 \\
\hline Videogame - Sem (min/dia) & 192 & $0^{*}$ & 30,0 & 187 & 30 & 60,0 \\
\hline Videogame - Fds (min/dia) & 198 & $0^{*}$ & 30,0 & 187 & 60 & 180,0 \\
\hline TT - Sem (min/dia) & 182 & $180^{*}$ & 180,0 & 182 & 210 & 180,0 \\
\hline TT -Fds(min/dia) & 184 & $240^{*}$ & 240,0 & 171 & 300 & 270,0 \\
\hline \multirow[t]{2}{*}{$\mathrm{TT}$ (min/dia) } & 175 & $195 *$ & 165,0 & 171 & 240 & 240,0 \\
\hline & $\mathrm{n}$ & Média & Desvio-padrão & $\mathrm{n}$ & Média & Desvio-padrão \\
\hline \multicolumn{7}{|l|}{ Antropometria } \\
\hline Estatura (m) & 301 & 1,4 & 0,1 & 274 & 1,4 & 0,1 \\
\hline \multicolumn{7}{|l|}{ Bioquímicas } \\
\hline GLI (mg/dL) & 242 & $79,8^{\#}$ & 9,6 & 203 & 81,8 & 8,6 \\
\hline
\end{tabular}


Tabela 2. Distribuição das variáveis antropométricas e número de repetição no teste de sentar em levantar em relação a condição de dependência para realização de atividades básicas da vida diária, Uberaba, MG, Brasil, 2010.

\begin{tabular}{|c|c|c|}
\hline \multirow{3}{*}{ Variáveis } & \multicolumn{2}{|c|}{ Comportamento sedentário } \\
\hline & \multicolumn{2}{|c|}{ Análise bruta } \\
\hline & RP (IC 95\%) & $p$-valor \\
\hline \multicolumn{3}{|l|}{ Bloco 1 - Aspectos sociodemográficos } \\
\hline Sexo & & 0,543 \\
\hline Masculino & 1 & \\
\hline Feminino & $1,037(0,903-1,164)$ & \\
\hline Idade cronológica & $1,103(1,002-1,214)$ & 0,292 \\
\hline Classe econômica & & 0,435 \\
\hline C, D e E & 1 & \\
\hline A e B & $0,924(0,758-1,127)$ & \\
\hline \multicolumn{3}{|l|}{ Bloco 2 - Aspectos de saúde } \\
\hline Índice de massa corporal (kg/m2) & & 0,909 \\
\hline Sobrepeso e obesidade & 1 & \\
\hline Eutrófico & $1,010(0,846-1,207)$ & \\
\hline Classificação gordura corporal & & 0,964 \\
\hline Alto & 1 & \\
\hline Ótimo & $0,997(0,877-1,133)$ & \\
\hline Síndrome metabólica & & 0,415 \\
\hline SM & 1 & \\
\hline Não presença de SM & $0,925(0,767-1,116)$ & \\
\hline \multicolumn{3}{|l|}{ Bloco 3 - Aspectos comportamentais } \\
\hline Recomendação AF & & 0,994 \\
\hline$<60 \mathrm{~min} / \mathrm{dia}$ & 1 & \\
\hline$\geq 60 \mathrm{~min} / \mathrm{dia}$ & $1,000(0,886-1,130)$ & \\
\hline Deslocamento para escola & & 0,746 \\
\hline 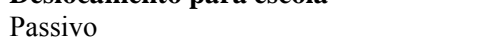 & 1 & \\
\hline Ativo & $0,976(0,845-1,128)$ & \\
\hline Tempo de sono por noite & & 0,809 \\
\hline Menos que $8 \mathrm{~h}$ & 1 & \\
\hline Mais de $8 \mathrm{~h}$ & $1,019(0,878-1,182)$ & \\
\hline Frequenta escolinha esportiva & & 0,763 \\
\hline Não & 1 & \\
\hline Sim & $0,971(0,799-1,178)$ & \\
\hline
\end{tabular}

sociodemográficas, de saúde e comportamentais. A Tabela 2 expõe os valores de RP, intervalo de confiança e $p$-valor encontrados nas análises bivariadas.

\section{Discussão}

O presente estudo investigou fatores associados ao CS em escolares de 9-12 anos de idade do município de UberabaMG. Meninas apresentaram valores significativamente superiores para dobras cutâneas, triglicerídeos e HDL-c. Meninos apresentaram maiores valores de glicemia, atenderam mais à recomendação de $60 \mathrm{~min}$ de AFMV/dia e, ao mesmo tempo, apresentaram maior CS, tanto no TT durante a semana e final de semana, como no tempo dedicado ao videogame. No modelo de regressão de Poisson, variáveis sociodemográficas, de saúde e comportamentais não foram associadas ao CS.

$\mathrm{O}$ presente estudo não encontrou associação entre IMC e CS, corroborando com os resultados recentes apresentados por Byun, Liu e Pate (2013), que analisaram dois estudos (n $=418$ ) com pré-escolares utilizando acelerometria. Esperavase que obesos apresentassem combinação de exposição excessiva ao TT e baixos níveis de AF (Anderson, Economos, \& Must, 2008; Danielsen et al., 2011; Harrison et al., 2006; Olds, Ridley, \& Dollman, 2006; Pearce, Basterfield, Mann, Parkinson, \& Adamson, 2012). Por outro lado, crianças e adolescentes que praticam $\mathrm{AF}$ regular podem diminuir o risco de sobrepeso e obesidade (Colley et al., 2012; Machadorodrigues et al., 2012; Mushtaq et al., 2011), ou seja, o tempo gasto em CS por obesos e não obesos pode não apresentar diferença significativa, contudo estes parecem compensar o TT com participação significativamente maior em AFMV (Hughes et al., 2006). Assim, programas de intervenção para prevenção de obesidade infantil devem envolver, além do aumento da prática de AF, habitualmente preconizado, também a redução da exposição ao CS, aqui caracterizado como TT.

O TT estimula hábitos dietéticos inadequados, comportamentos agressivos e abuso de determinadas substâncias devido às mensagens transmitidas em programas e publicidade (Hancox, Milne, \& Poulton, 2004), com evidências de que tais hábitos aumentam tanto a ingestão calórica diária total, quanto a ingestão diária de gorduras (Gore, Foster, DiLillo, Kirk, \& Smith West, 2003).

Embora seja o indicador mais utilizado para classificação 
do sobrepeso e obesidade em estudos epidemiológicos, o IMC pode não captar a variação na composição corporal durante a adolescência. Contudo, assim como IMC, excesso de gordura corporal não foi associado ao CS. Espera-se que quanto maior o tempo dedicado ao CS maior a quantidade de gordura corporal (Pelegrini \& Petroski, 2008). Contudo, as meninas apresentaram valores significativamente maiores de gordura corporal, explicado pela influência do estrogênio, que apesar de aumentar a taxa metabólica, corresponde a um terço do aumento produzido pela testosterona nesta fase da vida (Guyton \& Hall, 1997). Estes achados corroboram recente revisão, a qual constatou que associação entre CS e ganho de peso ainda não são conclusivos (Froberg \& Andersen, 2005).

A SM não diferiu significativamente entre os sexos e não apresentou associação com CS. Contudo, merece atenção a prevalência de $12,3 \%$ da SM na amostra. A identificação precoce da SM em crianças e adolescentes é importante, a fim de evitar a morbi-mortalidade que geralmente é causada por esta síndrome. Contudo, a avaliação dos fatores de risco para SM exige valores apropriados para diferentes idades e sexos (Saffari, Jalilolghadr, Esmailzadehha, \& Azinfar, 2012) e, dessa forma, necessita definição padronizada e internacionalmente aceita para seu diagnóstico, pois as prevalências de SM em crianças e adolescentes variam não só por causa de diferentes populações estudadas, como também pela adoção de definições diferentes (Andaki, Tinoco, Mendes, \& Amorim, 2012).

CS vem sendo associado à biomarcadores de doenças cardiovasculares e metabólicas, bem como a fatores de risco como PC, PA e dislipidemias (Della Manna et al., 2006; Edwardson et al., 2012). Danielsen et al. (2011) encontraram associação significativa entre TT e HOMA-IR, independentes da AF e do consumo de gordura e açúcar em crianças. Mais ainda, crianças e adolescentes com $\mathrm{TT} \geq 4$ horas/dia apresentam 2,53 vezes mais chances de apresentar fatores de risco cardiometabólicos do que aqueles que assistiam $<1$ hora/dia (Carson \& Janssen, 2011). Em contraste, a AF foi significativa e inversamente associada ao conjunto de fatores de risco metabólico, independente do TT e adiposidade (Ekelund et al., 2006). Mesmo independente de gordura corporal, o estilo de vida ativo proporciona atividade contrátil de músculos que podem estimular alguns processos bioquímicos e moleculares importantes para prevenir os efeitos do CS em doenças crônicas metabólicas (Freedman et al., 2008).

AF não apresentou associação com CS e, dessa forma, deve ser tratado como construtos independentes (Aggio et al., 2012; Ekelund et al., 2006; Ford \& Li, 2008; Kang et al., 2010; Pate et al., 2011; Pearce et al., 2012; Sandercock \& Ogunleye, 2012). Crianças podem apresentar TT excessivo durante o dia e, ao final dele, frequentar escolinha esportiva ou brincar ao ar livre. Além disso, o uso de mídias eletrônicas coexiste entre indivíduos com diferentes níveis de AF. AF diferiu significativamente entre os sexos, onde os meninos, ao mesmo tempo, alcançam as recomendações de $60 \mathrm{~min} / \mathrm{dia}$ (Anderson et al., 2008; Hopkins et al., 2012; Hume et al.,
2012; King et al., 2011; Oliveira, Silva, Santos, Silva, \& Conceição, 2010) e apresentam exposição excessiva ao TT (Aires et al., 2011; Leatherdale, Faulkner, \& Arbournicitopoulos, 2010).

Com relação aos aspectos socioeconômicos o presente estudo demonstrou que escolares pertencentes às classes econômicas A e B permaneceram tempo significativamente maior em CS do que aqueles das classes C, D e E (Mushtaq et al., 2011; Olds et al., 2006; Oliveira et al., 2010). Por outro lado, Anderson et al. (2008) não encontraram associações entre classe econômica e TT ou, ainda, encontrou-se associação inversa (Kang et al., 2010; Pate et al., 2011; Tandon et al., 2012). Desse modo, os achados sobre a associação entre CS e classe econômica ainda se encontram inconsistentes. Por exemplo, crianças pertencentes à classe econômica privilegiada podem ter mais acesso às novas tecnologias e, consequentemente, aumentar seu TT, ou crianças de classe econômica mais baixa podem gastar seu tempo livre frente à TV ou frequentar horas de lan houses. O TT encontrado foi maior entre os meninos $(p=0,045)$, de acordo com outros achados na literatura (Aires et al., 2011; Barr-Anderson et al., 2011; Leatherdale et al., 2010; Mushtaq et al., 2011; Olds et al., 2006). Porém, outros estudos não encontraram diferença significativa entre sexos para o TT (Anderson et al., 2008; Hume et al., 2012), ou mesmo reportaram meninas com maior TT (King et al., 2011; Pearce et al., 2012). De maneira geral, meninos jogam mais videogame e meninas utilizam mais o computador (Olds et al., 2006) e esta variabilidade pode ser explicada, em parte, pelos diferentes instrumentos de medidas e metodologias aplicadas. Por exemplo, CS como resultado do somatório de exposição frente à TV, computador e videogame, ou apenas exposição à TV (Lubans et al., 2011; Pate et al., 2011; ReyLópez, Vicente-Rodríguez, Biosca, \& Moreno, 2008; Tremblay, Leblanc, Kho, et al., 2011). O entendimento do tipo de exposição à tela merece esclarecimentos com intuito de colaborar com intervenções sexo dirigidas.

O CS foi prevalente em 76,9 \% da amostra (TT $\geq 2 \mathrm{~h}$ ). Valores de IMC, PC, PAS e triglicerídeos podem apresentar maiores alterações de acordo com o aumento do quartil de $\mathrm{TT}$, assim crianças e adolescentes com $\mathrm{TT} \geq 7 \mathrm{~h}$ apresentam 2,88 mais chances para SM (Kang et al., 2010) e meninos com TT $<2 \mathrm{~h}$ apresentam o dobro de chances de apresentar boa aptidão cardiorrespiratória do que aqueles com TT $>4 \mathrm{~h}$ (Sandercock \& Ogunleye, 2012). Assim, prejuízos à saúde associados ao prolongado TT tornam-se evidentes em limiares mais elevados.

A exposição ao TT foi maior nos finais de semana do que durante a semana, de acordo com alguns achados na literatura (Devís-Devís, Peiro-Velert, Beltran-Carrillo, \& Tomas, 2009; Ewalt, Danduran, Strath, Moerchen, \& Swartz, 2011; Olds et al., 2006), talvez devido ao maior tempo livre preenchido pela facilidade à acessibilidade à tecnologia doméstica (Devís-Devís et al., 2009; Pate et al., 2011). O TT para um dia de fim de semana está fortemente associado (OR $=2,62,95 \%$ CI: 1,16 - 5,90) à SM (Kang et al., 2010), mas não nos dias de semana. Intervenções devem ser direcionadas à 
redução do CS, principalmente durante o fim de semana de forma a minimizar o risco para SM.

A forma de deslocamento para a escola não apresentou associação com CS. Contudo, este construto é merecedor de atenções, pois contribui para o gasto calórico diário total (Tudor-Locke, Ainsworth, \& Popkin, 2001), e tem sido associado positivamente ao nível geral de AF (Lee, Orenstein, \& Richardson, 2008). O deslocamento passivo para a escola foi associado ao CS e constitui fator comportamental potencialmente modificável (King et al., 2011). Para promover a diminuição do CS e obter benefícios à saúde, o deslocamento ativo para escola parece ser uma boa alternativa. Recentes investigações têm reportado que o transporte ativo está associado ao aumento do tempo dedicado à $\mathrm{AF}$ (King et al., 2011), melhor aptidão cardiorrespiratória (Sandercock \& Ogunleye, 2012), menor propensão ao excesso de peso (Mushtaq et al., 2011), PC normal e adequados níveis de HDL-c (Pizarro, Ribeiro, Marques, Mota, \& Santos, 2013). Assim, o transporte ativo para escola deve ser considerado, pois contribui para o ajuste da criança às recomendações de $\mathrm{AF}$ e CS.

Torna-se evidente a importância de investigações e intervenções que visem à diminuição do CS ainda na infância focadas na promoção da saúde e qualidade de vida até a idade adulta. Veerman et al. (2012) reportaram que, após 25 anos de idade, cada hora frente à TV reduz a expectativa de vida em 21,8 min, ou seja, pessoas que passam $6 \mathrm{~h} /$ dia frente à TV possuem aproximadamente 4,8 anos a menos de expectativa de vida, quando comparadas a pessoas que não assistem TV diariamente.

As intervenções sugeridas para promover alterações nesse comportamento nocivo são modificações no ambiente, remover aparelhos eletrônicos do quarto de crianças, restringir o acesso à televisão ou ao computador utilizando controladores de tempo nos dispositivos e oferecendo mais oportunidades de atividade física às crianças (Maniccia, Davison, Marshall, Manganello, \& Dennison, 2011).

$\mathrm{O}$ presente estudo investigou fatores associados ao CS em escolares brasileiros, porém com algumas limitações. Este foi um estudo transversal, assim torna-se incapaz de determinar causalidade, porém este delineamento oferece resposta rápida sobre o tema investigado e pode auxiliar futuros estudos e intervenções a formularem hipóteses e estratégias. AF e CS foram estimados por meio de questionários, que apresentam vieses de aplicação e recordação por parte do entrevistador e entrevistado, respectivamente. Além disso, medidas auto-relatadas de comportamentos socialmente indesejáveis, tais como assistir $\mathrm{TV}$, usar computador, não praticar $\mathrm{AF}$, tendem a ser subestimados. Valores de CS variam entre os relatos dos pais e medida direta, por acelerometria (Colley et al., 2012). Recente estudo verificou que o tempo sentado auto-relato não está associado com a incidência de doenças cardiovasculares em mulheres de meia idade (Herber-Gast, Jackson, Mishra, \& Brown, 2013). Contudo, ainda é o método mais utilizado por questões logísticas, principalmente em países como o Brasil (Hallal, Matsudo, \& Farias, 2012). A definição do CS e suas consequências podem estar relacionadas com os desafios de sua medição. A heterogeneidade nos dados de TT torna desafiador à interpretação e utilização dos resultados encontrados nos estudos (Wahi, Parkin, Beyene, Uleryk, \& Birken, 2011).

O CS não foi associado a variáveis sociodemográficas, de saúde e comportamentais em crianças de 9-12 anos de idade. Estes resultados são o contrário das expectativas, dada a crescente evidência de uma relação entre tempo sentado e doenças crônicas não transmissíveis, como Mais pesquisas são necessárias para elucidar as inter-relações complexas entre TT e obesidade, síndrome metabólica, hipertensão arterial para confirmar ou refutar essas conclusões, principalmente entre crianças e adolescentes

\section{Referências}

Aggio, D., Ogunleye, A. A., Voss, C., \& Sandercock, G. R. H. (2012). Temporal relationships between screen- time and physical activity with cardiorespiratory fitness in English Schoolchildren: A 2-year longitudinal study. Preventive Medicine, 55(1), 37-39.

Aires, L., Pratt, M., Lobelo, F., Santos, R. M., Santos, M. P., \& Mota, J. (2011). Associations of cardiorespiratory fitness in children and adolescents with physical activity, active commuting to school, and screen time. Journal of Physical Activity \& Health, 8 Suppl 2, S198.

Andaki, A. C. R. (2010). Predição da síndrome metabólica em crianças por meio das medidas antropométricas e nível de atividade física. Universidade Federal de Viçosa, Viçosa, MG.

Andaki, A. C. R., Tinoco, A. L. A., Mendes, E. L., \& Amorim, P. R. S. (2012). Diagnostics Criteria of Metabolic Syndrome in Children. In C. M. L. Garcia \& P. A. P. Gonzalez (Eds.), Handbook on Metabolic Syndrome: Classification, Risk Factors and Health Impact (1 ed., Vol. 1, pp. 305-317). New York: Nova Publishers.

Anderson, S., Economos, C., \& Must, A. (2008). Active play and screen time in US children aged 4 to 11 years in relation to sociodemographic and weight status characteristics: a nationally representative cross-sectional analysis. BMC Public Health, 8(1), 366.

Associação Brasileira de Empresas de Pesquisa. (2011). Dados com base no Levantamento Sócio Econômico 2009 - IBOPE.

Barr-anderson, D. J., Fulkerson, J. A., Smyth, M., Himes, J. H., Hannan, P. J., Holy Rock, B., \& Story, M. (2011). Associations of American Indian children's screen- time behavior with parental television behavior, parental perceptions of children's screen time, and media-related resources in the home. Preventing Chronic Disease, 8(5), A105.

Byun, W., Liu, J., \& Pate, R. R. (2013). Association between objectively measured sedentary behavior and body mass index in preschool children. [Journal article]. International Journal of Obesity, 15(10), 222.

Carson, V., \& Janssen, I. (2011). Volume, patterns, and types of sedentary behavior and cardio-metabolic health in children and adolescents: a cross-sectional study. BMC Public Health, 11, 274.doi:1471-2458-11-274[pii],10.1186/1471-2458-11-274

Cole, T. J., Bellizzi, M. C., Flegal, K. M., \& Dietz, W. H. (2000). Establishing a standard definition for child overweight and obesity worldwide: international survey. BMJ, 320(7244), 1240-1243. 
Colley, R. C., Wong, S. L., Garriguet, D., Janssen, I., Gorber, S. C., \& Tremblay, M. S. (2012). Physical activity, sedentary behaviour and sleep in Canadian children: parent-report versus direct measures and relative associations with health risk. Health reports/Statistics Canada, Canadian Centre for Health Information = Rapports sur la santé/Statistique Canada, Centre canadien d'information sur la santé, 23(2), 45.

Danielsen, Y. S., Júlíusson, P. B., Nordhus, I. H., Kleiven, M., Meltzer, H. M., Olsson, S. J. G., \& Pallesen, S. (2011). The relationship between life-style and cardio-metabolic risk indicators in children: the importance of screen time. Acta paediatrica (Oslo, Norway:1992), 100(2), 253.

de Ferranti, S. D., Gauvreau, K., Ludwig, D. S., Neufeld, E. J., Newburger, J. W., \& Rifai, N. (2004). Prevalence of the metabolic syndrome in American adolescents: findings from the Third National Health and Nutrition Examination Survey. Circulation, 110(16), 2494-2497. doi: 01.CIR.000 0145117.40114.C7 [pii], 10.1161/01.CIR.00001451 17.40114.C7

Della Manna, T., Damiani, D., \& Setian, N. (2006). Síndrome metabólica: revisão. Pediatria, 28(4), 272-277.

Department of Health. (2004). At least five a week: evidence on the impact of physical activity and its relationship to health. London, UK: Department of Health,

Devís-Devís, J., Peiro-Velert, C., Beltran-Carrillo, V. J., \& Tomas, J. M. (2009). Screen Media Time Usage of 12-16 Year-Old Spanish School Adolescents: Effects of Personal and Socioeconomic Factors, Season and Type of Day. Journal of Adolescence, 32(2), 213-231.

Edwardson, C. L., Gorely, T., Davies, M. J., Gray, L. J., Khunti, K., Wilmot, E. G., . . B Biddle, S. J. (2012). Association of sedentary behaviour with metabolic syndrome: a meta-analysis. PLoS One, 7(4), e34916. doi: 10.1371/journal.p one.0034916 [doi], PONE-D-11-16815 [pii].

Ekelund, U., Brage, S., Froberg, K., Harro, M., Anderssen, S. A., Sardinha, L. B., . . . Andersen, L. B. (2006). TV Viewing and Physical Activity Are Independently Associated with Metabolic Risk in Children: The European Youth Heart Study. [doi:10.1371/journal.pmed.0030488]. PLoS Med, 3(12), e488.

Ewalt, L. A., Danduran, M. J., Strath, S. J., Moerchen, V., \& Swartz, A. M. (2011). Objectively assessed physical activity and sedentary behaviour does not differ between children and adolescents with and without a congenital heart defect: a pilot examination. Cardiology in the young, 22(1), 34.

Ford, E. S., \& Li, C. (2008). Defining the metabolic syndrome in children and adolescents: will the real definition please stand up? The Journal of pediatrics, 152(2), 160-164.

Freedman, D. S., Patel, D. A., Srinivasan, S. R., Chen, W., Tang, R., Bond, M. G., \& Berenson, G. S. (2008). The contribution of childhood obesity to adult carotid intima-media thickness: the Bogalusa Heart Study. International Journal of Obesity (Lond), 32(5), 749-756. doi: 0803798 [pii], 10.1038/ sj.ijo.0803798 [doi].

Froberg, K., \& Andersen, L. B. (2005). Mini review: physical activity and fitness and its relations to cardiovascular disease risk factors in children. International Journal of Obesity (Lond), 29 Suppl 2, S34-39.

Gore, S. A., Foster, J. A., DiLillo, V. G., Kirk, K., \& Smith West, D. (2003). Television viewing and snacking. Eating Behavior, 4(4), 399-405. doi: 10.1016/s1471-0153(03)00053-9.

Guyton, A. C., \& Hall., J.E. (1997). Tratado de Fisiologia Médica. Funções reprodutoras e hormônios masculinos. Cap.80 (9 ed. ed.). Rio de Janeiro: Guanabara Koogan.
Hallal, P. C., Matsudo, S., \& Farias, J. C., Jr. (2012). Measurement of physical activity by self-report in low- and middle-income countries: more of the same is not enough. Journal of Physical Activity and Health, 9(1), S88-90.

Hancox, R. J., Milne, B. J., \& Poulton, R. (2004). Association between child and adolescent television viewing and adult health: a longitudinal birth cohort study. The Lancet, 364(9430), 257-262. doi: http://dx.doi.org/10.1016/S01406736(04)16675-0.

Hardy, L. L., Dobbins, T. A., Denney-Wilson, E. A., Okely, A. D., \& Booth, M. L. (2009). Sedentariness, small-screen recreation, and fitness in youth. American Journal of Preventive Medicine, 36(2), 120-125. doi: S0749-3797(08)00870-2 [pii], 10.1016/ j.amepre.2008.09.034.

Harrison, M., Burns, C. F., McGuinness, M., Heslin, J., \& Murphy, N. M. (2006). Influence of a health education intervention on physical activity and screen time in primary school children: 'Switch Off--Get Active'. Journal of Science and Medicine in Sport/Sports Medicine Australia, 9(5), 388.

Herber-Gast, G.-C., Jackson, C., Mishra, G., \& Brown, W. (2013). Self-reported sitting time is not associated with incidence of cardiovascular disease in a population-based cohort of midaged women. International Journal of Behavioral Nutrition and Physical Activity, 10(1), 55.

Hopkins, N., Stratton, G., Ridgers, N. D., Graves, L. E. F., Cable, N. T., \& Green, D. J. (2012). Lack of relationship between sedentary behaviour and vascular function in children. European Journal of Applied Physiology, 112(2), 617.

Hughes, A. R., Henderson, A., Ortiz - Rodriguez, V., Artinou, M. L., Reilly, J. J., \& Ortiz-Rodriguez, V. (2006). Habitual physical activity and sedentary behaviour in a clinical sample of obese children. International Journal of Obesity, 30(10), 1494.

Hume, C., Salmon, J., Veitch, J., O'Connell, E., Crawford, D., \& Ball, K. (2012). Socio-demographic characteristics of children experiencing socioeconomic disadvantage who meet physical activity and screen- time recommendations: the READI study. Preventive Medicine, 54(1), 61.

Kang, H.-T., Lee, H.-R., Shim, J.-Y., Shin, Y.-H., Park, B.-J., \& Lee, Y.-J. (2010). Association between screen time and metabolic syndrome in children and adolescents in Korea: The 2005 Korean National Health and Nutrition Examination Survey. Diabetes Research and Clinical Practice, 89(1), 72-78.

King, A. C., Parkinson, K. N., Adamson, A. J., Murray, L., Besson, H., Reilly, J. J., \& Basterfield, L. (2011). Correlates of objectively measured physical activity and sedentary behaviour in English children. [Research Support, Non-U.S. Gov't]. European Journal of Public Health, 21(4), 424-431. doi: 10.1093/eurpub/ckq104.

Leatherdale, S. T., Faulkner, G., \& Arbour-nicitopoulos, K. (2010). School and Student Characteristics Associated With ScreenTime Sedentary Behavior Among Students in Grades 5-8, Ontario, Canada, 2007-2008. Preventing Chronic Disease, 7(6).

Lee, M. C., Orenstein, M. R., \& Richardson, M. J. (2008). Systematic review of active commuting to school and childrens physical activity and weight. Journal of Physical Activity and Health, 5(6), 930-949.

Lohman, T. G. (1987). The Use of Skinfold to Estimate Body Fatness on Children and Youth. Journal of Physical Education, Recreation \& Dance, 58(9), 98-102.

Lohman, T. G., Roche, A. F., \& Martorell, R. (1988). Anthropometric standardization reference manual. Champaign, IL: Human Kinetics Books.

Lubans, D. R., Hesketh, K., Cliff, D. P., Barnett, L. M., Salmon, 
J., Dollman, J., . . Hardy, L. L. (2011). A systematic review of the validity and reliability of sedentary behaviour measures used with children and adolescents. Obesity reviews: an Official Journal of the International Association for the Study of Obesity, 12(10), 781 .

Machado-rodrigues, A. M., Coelho-e-silva, M. J., Mota, J., Padez, C., Ronque, E., Cumming, S. P., \& Malina, R. M. (2012). Cardiorespiratory fitness, weight status and objectively measured sedentary behaviour and physical activity in rural and urban Portuguese adolescents. Journal of Child Health Care: for professionals working with children in the hospital and community, 16(2), 166.

Maniccia, D. M., Davison, K. K., Marshall, S. J., Manganello, J. A., \& Dennison, B. A. (2011). A meta-analysis of interventions that target children's screen time for reduction. Pediatrics, 128(1), e193-210. doi: 10.1542/peds.2010-2353

Mushtaq, M. U., Gull, S., Mushtaq, K., Shahid, U., Shad, M. A., \& Akram, J. (2011). Dietary behaviors, physical activity and sedentary lifestyle associated with overweight and obesity, and their socio-demographic correlates, among Pakistani primary school children. The International Journal of Behavioral Nutrition and Physical Activity, 8, 130-130.

Nobre, F. (2010). [Introduction: Brazilian guidelines on hypertension VI. Brazilian Society of Cardiology, Brazilian Society of Hypertension, Brazilian Society of Nephrology]. [Introductory]. Jornal Brasileiro de Nefrologia, 32 Suppl 1, III.

Olds, T., Ridley, K., \& Dollman, J. (2006). Screenieboppers and extreme screenies: the place of screen time in the time budgets of 10-13 year?old Australian children (Vol. 30, pp. 137-142). Oxford, UK.

Oliveira, T. C. d., Silva, A. A. M. d., Santos, C. d. J. N. d., Silva, J. S. e., \& Conceição, S. I. O. d. (2010). Physical activity and sedentary lifestyle among children from private and public schools in Northern Brazil. Revista de Saúde Pública, 44, 9961004 .

Pate, R. R., Mitchell, J. A., Byun, W., \& Dowda, M. (2011). Sedentary behaviour in youth. British Journal of Sports Medicine, 45(11), 906-913. doi: 45/11/906 [pii], 10.1136/ bjsports-2011-090192 [doi].

Pate, R. R., O'Neill, J. R., \& Lobelo, F. (2008). The evolving definition of "sedentary". Exercise Sport Science Review, 36(4), 173-178. doi: 10.1097/JES.0b013e3181877d1a, 00003677 200810000-00002 [pii].

Pearce, M. S., Basterfield, L., Mann, K. D., Parkinson, K. N., \& Adamson, A. J. (2012). Early predictors of objectively measured physical activity and sedentary behaviour in 8-10 year old children: the gateshead millennium study. PLoS One, 7(6), e37975.

Pelegrini, A., \& Petroski, E. L. (2008). Relação entre o tempo em frente à $\mathrm{TV}$ eo gasto calórico em adolescentes com diferentes percentuais de gordura corporal. Revista Brasileira de Cineantropometria e Desempenho Humano, 10(1).

Pizarro, A., Ribeiro, J., Marques, E., Mota, J., \& Santos, M. (2013). Is walking to school associated with improved metabolic health?: a cross sectional study in 10 to 12 year old Portuguese children. International Journal of Behavioral Nutrition and Physical Activity, 10(1), 12.

Rey-López, J. P., Vicente-Rodríguez, G., Biosca, M., \& Moreno, L. A. (2008). Sedentary behaviour and obesity development in children and adolescents. Nutrition, Metabolism and Cardiovascular Diseases, 18(3), 242-251.

Saffari, F., Jalilolghadr, S., Esmailzadehha, N., \& Azinfar, P. (2012).
Metabolic syndrome in a sample of the 6- to 16-year-old overweight or obese pediatric population: a comparison of two definitions. The Clinincal and Risk Management, 8, 5563. doi: 10.2147/TCRM.S26673, tcrm-8-055 [pii].

Sallis, J. F., Strikmiller, P. K., Harsha, D. W., Feldman, H. A., Ehlinger, S., Stone, E. J., . . . Woods, S. (1996). Validation of interviewer- and self-administered physical activity checklists for fifth grade students. Medicine and Science in Sports and Exercise, 28(7), 840-851.

Sandercock, G. R. H., \& Ogunleye, A. A. (2012). Screen time and passive school travel as independent predictors of cardiorespiratory fitness in youth. Preventive Medicine, 54(5), 319-322.

Sardinha, L. B., \& Magalhães, J. (2012). Comportamento Sedentário. Revista Factores de Risco(27), 54-64.

Slaughter, M. H., Lohman, T. G., Boileau, R. A., Horswill, C. A., Stillman, R. J., Van Loan, M. D., \& Bemben, D. A. (1988). Skinfold equations for estimation of body fatness in children and youth. Human Biology, 60(5), 709-723.

Tandon, P. S., Zhou, C., Sallis, J. F., Cain, K. L., Frank, L. D., \& Saelens, B. E. (2012). Home environment relationships with children's physical activity, sedentary time, and screen time by socioeconomic status. The International Journal of Behavioral Nutrition and Physical Activity, 9, 88.

Tavares, L. F., Yokoo, E. M., Rosa, M. L. G., \& Fonseca, S. C. (2010). Síndrome metabólica em crianças e adolescentes brasileiros: revisão sistemática. Caderno de Saúde Coletiva [Internet], 18(4), 469-476.

te Velde, S. J., van Nassau, F., Uijtdewilligen, L., van Stralen, M. M., Cardon, G., De Craemer, M., . . Chinapaw, M. J. (2012). Energy balance-related behaviours associated with overweight and obesity in preschool children: a systematic review of prospective studies. Obesity Review, 13 Suppl 1, 56-74. doi: 10.1111/j.1467-789X.2011.00960.x.

Tremblay, M. S., Leblanc, A. G., Janssen, I., Kho, M. E., Hicks, A., Murumets, K., . . Duggan, M. (2011). Canadian sedentary behaviour guidelines for children and youth/Directives canadiennes en matiere de comportement sedentaire a l'intention des enfants et des jeunes.(Report). Applied Physiology, Nutrition, and Metabolism, 36(1), 59.

Tremblay, M. S., Leblanc, A. G., Kho, M. E., Saunders, T. J., Larouche, R., Colley, R. C., ... Gorber, S. C. (2011). Systematic review of sedentary behaviour and health indicators in schoolaged children and youth.(Review)(Report). The International Journal of Behavioral Nutrition and Physical Activity, 8, 98.

Tudor-Locke, C., Ainsworth, B. E., \& Popkin, B. M. (2001). Active commuting to school: an overlooked source of childrens' physical activity? Sports Med, 31(5), 309-313.

U.S. Department of Health and Human Services. (2008). Physical activity guidelines for Americans. The Oklahoma Nurse, 53(4), 25 .

Veerman, J. L., Healy, G. N., Cobiac, L. J., Vos, T., Winkler, E. A. H., Owen, N., \& Dunstan, D. W. (2012). Television viewing time and reduced life expectancy: a life table analysis. British Journal of Sports Medicine, 46(13), 927-930. doi: 10.1136/ bjsports-2011-085662.

Wahi, G., Parkin, P. C., Beyene, J., Uleryk, E. M., \& Birken, C. S. (2011). Effectiveness of Interventions Aimed at Reducing Screen Time in Children: A Systematic Review and Metaanalysis of Randomized Controlled Trials.(Report). Archives of Pediatrics \& Adolescent Medicine, 165(11), 979.

World Health Organization. (2010). Global recommendations on physical activity for health. Geneva: World Health Organization. 


\section{Nota dos autores}

\section{Correspondência}

Amanda Santos

Rua Quintino Bocaiúva, 637, Centro

Elói Mendes, Minas Gerais, 37.110.000.

Fone: (34) 9196-1696.

E-mail: amanda637@hotmail.com

Agradecimento à Fundação de Apoio a Pesquisa de Minas Gerais/ FAPEMIG e ao Programa de Bolsas REUNI.

Informação adicional: Este estudo é resultado de dissertação de mestrado.

Artigo completo de trabalho apresentado no VIII Congresso Internacional de Educação Física e Motricidade Humana e XIV Simpósio Paulista de Educação Física.

Declaração de Conflito de Interesses: Os autores declararam ausência de conflitos de interesse no que diz respeito à pesquisa, autoria e / ou publicação deste artigo.

Manuscrito recebido em 30 de março de 2013

Manuscrito aceito em 10 de maio de 2013 